\title{
Thermochromic Filters Effect on Static Light
}

Cabral, I.; Souto, A.P.

\begin{abstract}
Is it possible to change the environment light without changing or act in the light source? Smart textiles materials and new technologies open the possibility to introduce dynamic behaviours to elements that use to be static. Using the ability of Colour Change Materials to answer to external stimulus, this paper studies how colour variation of textile samples, treated with reversible thermochromic pigments, filter the artificial light intensity that go by (pass) through it.

In this work a set of screen printing samples have been developed in which have been applied thermochromic pigments or a mixture of thermochromic and common pigments. Differences in light transmission are measured and correlated with the different processes employed, an overlapping of layers of different pigments or only one layer with a mixture of both types of pigments, in such way to get similar colour when activated.

The results indicate that a sample activated by heat may achieve different light intensity transmitted, in terms of luminosity (dark colours absorb more than light colours) and according with the requirement of the pigments to absorb light to get colour.
\end{abstract}

